\title{
Single-Element Extensions of Matroids
}

\author{
Henry H. Crapo \\ Assistant Professor of Mathematics, Northeastern University, Boston, Mass. \\ (November 16, 1964)
}

\begin{abstract}
Extensions of matroids to sets containing one additional element are characterized in terms of modular cuts of the lattice of closed subsets. An equivalent characterization is given in terms of linear subclasses of the set of circuits or bonds of the matroid. A scheme for the construction of finite geometric lattices is derived and the existence of at least $2^{n}$ nonisomorphic matroids on an $n$-element set is established.
\end{abstract}

\section{Contents}

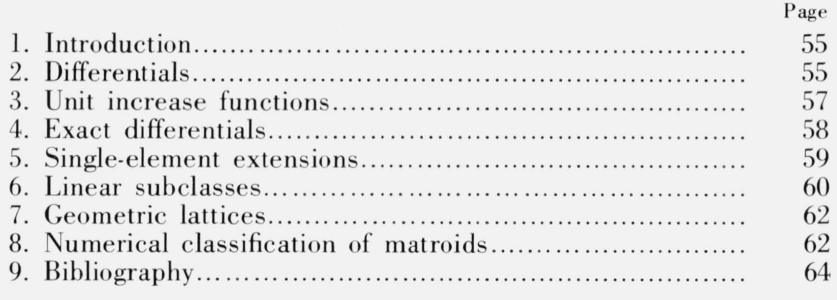

\section{Introduction ${ }^{1}$}

In order to facilitate inductive proofs of matroid ${ }^{2}$ theorems, we shall set forth a characterization of singleelement extensions of matroid structures. Our techniques are those developed in our recent paper, "Lattice Differentials and the Theory of Combinatorial Independence". Sufficient material is included in the next three sections to support the proofs of the extension theorems. The reader is referred to the above paper for details.

A matroid, defined on a finite set, may be thought of as a structure defined on a lattice, i.e., on the Boolean algebra of all subsets of the set. Not all the properties of Boolean algebras are used in the proof of matroid theorems, however. We have chosen modular lattices as the proper domain for our presentation of the material needed for extension theory.

\section{Differentials}

An element $y$ is said to cover an element $x$ in a lattice $L$ if $x<y$, and for any element $z, x<z \leqslant y$, implies $z=y$. A lattice $L$ is modular if, for all elements $x$ and $y$ in $L, x$ covers $x \wedge y$ if and only if $x \vee y$ covers $y$. A sequence $p: x=p_{0}<p_{1}<\ldots<p_{n}=y$

\footnotetext{
'The statement of the extension theorem and the classification of matroids are taken from the author's Ph.D. thesis, entitled "On the Theory of Combinatorial Independence", written under the supervision of Professor Gian-Carlo Rota, and presented to the Mass. Inst. of Technology, May 1964. We are grateful to Jack Edmonds, National Bureau of Standards, who pointed out the significance of these theorems and encouraged us in their presentation.

${ }^{2}$ H. Whitney, Amer. J., 1935.
}

of elements $p_{i}$ of a lattice $L$, in which $p_{i}$ covers $p_{i-1}$ for $i=1, \ldots ., n$, is a path (of length $n$ ) from $x$ to $y$. A step is a path of length 1 .

The partial order of the lattice induces an order on the set of steps of the lattice. If $[x, y]$ and $[u, w]$ are steps of a lattice $L$, we say $[x, y] \leqslant[u, w]$ if and only if $x=y \wedge u$ and $y \vee u=w$. If, in a modular lattice, a step $[u, w]$ covers a step $[x, y]$ in this ordering, then $[u, x]$ and $[y, w]$ are also steps.

Definition: A function $R$ defined for all steps $[x, y]$ of a finite modular lattice $L$, and taking values in the two-element lattice ${ }^{3}\{0,1\}$, is a differential if and only if it is

(a) Projective on steps: If steps $[x, y]$ and $[u, w]$ are in the order $[x, y] \leqslant[u, w]$, then $R[x, y] \geqslant R[u, w]$.

(b) Subadditive on steps: If a step $[u, w]$ covers a step $[x, y]$ then $R[x, y] \leqslant R[x, u] \vee R[u, w]$.

The local character of differentials may be represented pictorially. A local graph is any assignment of values 0 or 1 to the four steps of an interval $[x \wedge y$, $x \vee y]$ in a finite modular lattice $L$, where the elements $x$ and $y$ cover $x \wedge y$ and are thus covered by $x \vee y$.

Proposition: An assignment of values 0 and 1 to the steps of a finite modular lattice $\mathrm{L}$ determines a differential if and only if all local graphs are of the following five types: zero, mixed, prime, one, or inexact. (These types of local graph are defined as figure 1 . We indicate steps on which $R=1$ by double lines, $R=0$ by single lines.)

\footnotetext{
${ }^{3}$ Differentials of higher order may be obtained by allowing the range of the differential to be a lattice more general than $\{0,1\}$.
} 


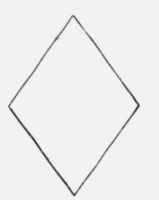

ZERO

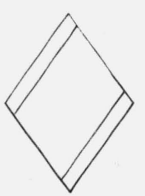

$M I X E D$
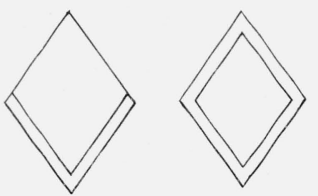

ONE

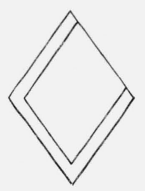

INEXACT
FIGURE 1

Proof: The projective and subadditive properties of differentials eliminate all local graphs except the five enumerated above. Conversely, if $R$ is a function on steps with values in the lattice $\{0,1\}$, and if all local graphs of $R$ are zero, mixed, prime, one, or inexact, the subadditive property follows, and the projective property is established, for steps $[x, y]$ and $[u, w]$, with $[x, y] \leqslant[u, w]$, as follows. Let $x=p_{0}<p_{1}$ $<\ldots .<p_{n}=u$ be a path from $x$ to $u$, and let $q=p \vee y$ be the projected path $y=p_{0} \vee y<p_{1} \vee y<\ldots<p_{n} \vee y$ $=w$ from $y$ to $w$. If $R[x, y]<R[u, w]$, then $R[x, y]=0$ and $R[u, w]=1$. Let $p_{i}$ be the greatest element such that $R\left[p_{i}, q_{i}\right]=0$. Then $R\left[p_{i+1}, q_{i+1}\right]=1$. Such a local graph $p_{i}, q_{i}, p_{i+1}, q_{i+1}$ is neither zero, mixed, prime, nor inexact.

A differential $R$ is termed exact if and only if it has no inexact local graph. Exact differentials are the subject of section 4 , below.

The three principal sources of differentials are closure operators, join-homomorphisms, and finite lattices. We describe these in turn.

If $C l$ is a closure operator $(x \leqslant C l(x)$, and $x \leqslant C l(y)$ implies $C l(x) \leqslant C l(y))$ on a finite modular lattice $L$,

$$
\begin{aligned}
R[x, y] & =1 \text { if } C l(x)<C l(y) \\
& =0 \text { otherwise }
\end{aligned}
$$

defines a differential $R$ on the steps of the lattice $L$.

A differential is canonically related to a unique closure on its domain lattice. We say an element $x$ of a finite modular lattice $L$ is closed relative to a differential $R$ on $L$ if and only if $R[x, y]=1$ for all steps. of the form $[x, y]$. We shall prove closed elements are elements $x$ such that $C l(x)=x$, in the closure $C l$ associated with $R$.

Proposition: Existence of Closed Elements: If $\mathrm{R}$ is a differential on a finite modular lattice $\mathrm{L}$, and if the differential $\mathrm{R}$ has value 1 on some step of $\mathrm{L}$, there exists a closed element $\mathrm{z}$ in $\mathrm{L}$ other than the element 1 .

Proof: Assume $R$ has value 1 on the step $[u, w]$, and say the lattice $L$ has height $n$. Choose a path $p$ from 0 to 1 which is maximal with respect to the number $k$ of initial steps on which the differential $R$ has value 0 . Then all steps beginning at $p_{k}$ must have differential value 1 , and $p_{k}$ is closed. It remains to prove that $k<n$, so that $p_{k}$ is not the uppermost element 1 of the lattice.

We prove by induction on the height $n$ of the lattice that a step $[u, w]$ on which $R$ has value 1 cannot coexist in $L$ with a path $p$ from 0 to 1 on which $R$ has value 0 . If $n=1: u=0$ and $w=1$, and no alternate path exists. Assume the truth of the statement for $n-1$, and let $L$ have height $n$. Since $L$ is modular, either $w \vee p_{n-1}=u \vee p_{n-1}$ and $w \wedge p_{n-1}$ covers $u \wedge p_{n-1}$, or else $w \vee p_{n-1}$ covers $u \vee p_{n-1}$ and $w \wedge p_{n-1}=u \wedge p_{n-1}$. In the first instance, we have the ordering of steps $\left[u \wedge p_{n-1}, w \wedge p_{n-1}\right] \leqslant[u, w]$, so $R\left[u \wedge p_{n-1}, w \wedge p_{n-1}\right]=1$ in the lattice interval $\left[0, p_{n-1}\right]$ of height $n-1$. This step cannot coexist with the portion of the path $p$ from 0 to $p_{n-1}$. In the second instance, choose a path $q$ from $u$ to $p_{n-1}$, and let $j$ be the greatest index for which $R\left[q_{j}, w \vee q_{j}\right]=1$. By subadditivity of $R$ on steps, $R\left[q_{j}, q_{j+1}\right]=1$, where $\left[q_{j}, q_{j+1}\right]$ is also a step in the interval sublattice $\left[0, p_{n-1}\right]$ of height $n-1$.

Proposition: Closures Formed from Differentials: Given a differential $\mathrm{R}$ on a finite modular lattice $\mathrm{L}$,

$$
\begin{aligned}
\mathrm{Cl}(\mathrm{x}) & =\sup \{\mathrm{y} ; \mathrm{H} \text { path from } \mathrm{x} \text { to y along which } \mathrm{R}=0\} \\
& =\inf \{\mathrm{z} ; \mathrm{z} \text { closed, } \mathrm{x} \leqslant \mathrm{z}\}
\end{aligned}
$$

is a closure operator on the lattice $\mathrm{L}$, the differential of which is $\mathrm{R}$.

PRoof: If an element $x$ is connected to two elements $y_{1}$ and $y_{2}$ by paths along which $R=0$, every step in a path from $y_{1}$ to $y_{1} \vee y_{2}$ exceeds, in the ordering on steps, some step in the path from $x$ to $y_{2}$. Thus $R=0$ on the extended path from $x$ to $y_{1} \vee y_{2}$, so $y_{1} \vee y_{2} \leqslant C l(x)$. If a path exists from $x$ to $y$ along which $R=0$, then $R$ must have value 0 on all steps of the lattice interval $[x, y]$, as was shown in the proof of the previous proposition. Thus the closure $C l(x)$ may be characterized as the maximum element $y$ such that $R=0$ everywhere on the lattice interval $[x, y]$. This element $C l(x)$ must be closed, for otherwise a path along which $K=0$ would exist from $x$ to an element covering $\operatorname{Cl}(x)$.

The set of closed elements of the lattice $L$ is closed with respect to the lattice operation inf. If $x$ and $y$ are closed elements, and an element $u$ covers $x \wedge y$, then $u \neq x$ or $u \neq y$. Say $u \neq y$. Then $u v y$ covers $y$, $[x \wedge y, u] \leqslant[y, u \vee y]$ in the ordering on steps, and $R[x \wedge y, u]=1$ because the element $y$ is closed and $R[y, u \vee y]=1$. Thus, for any element $x$ in $L, C l(x)$ may be characterized as the infimum of all closed elements $z$ such that $x \leqslant z$. As is proven in the theory of closure operators, ${ }^{4}$ any inf-closed class of lattice elements generates a closure operator by this rule.

It remains to prove that the differential of the closure operator $C l$ is the original differential $R$, i.e., that $R[x, y]=1$ if and only if $C l(x)<C l(y)$. If $R[x, y]=1$, there is a relatively closed element $z$ in the interval sublattice $[x, C l(y)]$ other than $C l(y)$. If an element $u$ covers $z$ in the lattice $L$, but $u \notin C l(y)$, then $u \vee C l(y)$ covers $C l(y), R[C l(y), u \vee C l(y)]=1$, and $R[z, u]=1$ because $R$ is projective on steps. Thus the element $z$ is closed, and $C l(x) \leqslant z<C l(y)$. Conversely, if $C l(x)<C l(y)$, then $y \vee C l(x)$ covers $C l(x)$ and $[x, y] \leqslant[C l(x), y \vee C l(x)]$ implies $R[x, y]=1$.

The set of closed elements of a differential $R$ on a finite modular lattice $L$, with partial ordering induced by that on $L$, forms a finite lattice $L / R$, called the

\footnotetext{
${ }^{4} \mathrm{O}$. Ore, Theory of Graphs.
} 
lattice of closed elements of $R$ on $L$. The meetirreducible elements (elements covered by exactly one other element) and join-irreducible elements (elements covering exactly one other element) figure prominently in the theory of differentials. Those meet-irreducible elements covered by 1 are called coatoms; those join-irreducible elements covering 0 are called atoms.

As a second source for differentials, consider join-homomorphisms $\quad(f(x \vee y)=f(x) \vee f(y))$ from a finite modular lattice $L$ into a lattice $Q$. A closure operator and a differential are induced on the domain of a join-homomorphism in much the same way that a partition is induced on the domain of a function.

Proposition: If $f$ is a join-homomorphism from a finite modular lattice $\mathrm{L}$ into a lattice $\mathrm{Q}$, then

$$
\begin{aligned}
& \mathrm{R}[\mathrm{x}, \mathrm{y}]=1 \text { if } \mathrm{f}(\mathrm{x})<\mathrm{f}(\mathrm{y}) \\
& \mathrm{R}[\mathrm{x}, \mathrm{y}]=0 \text { otherwise }
\end{aligned}
$$

defines a differential $\mathrm{R}$ on the steps $[\mathrm{x}, \mathrm{y}]$ of the lattice $\mathrm{L}$.

Proof: Assume steps $[x, y]$ and $[u, w]$ are in the order $[x, y] \leqslant[u, w]$. If $R[x, y]=0$, then $f(y)=f(x)$ $\leqslant f(u)$, because $x \leqslant u$ and $f$, being a join-homomorphism, is order-preserving. Thus $f(w)=l f(y \vee u)$ $=f(y) \vee f(u)=f(u)$, so $R[u, w]=0$, proving $R$ is projec tive on steps. If a step $[u, w]$ covers a step $[x, y]$, and if $R[x, u]=R[u, w]=0$, then $f(x) \leqslant f(y) \leqslant f(w)$ $=f(u)=f(x)$, so $f(x)=f(y)$, and $R[x, y]=0$, proving subadditivity.

Any differential may be represented as that of a join-homomorphism, by letting the lattice $Q$ be the Boolean algebra of all subsets of the set $M$ of meetirreducible elements of the lattice $L / R$, and by mapping each element $x \in L$ into the subset of $M$ composed of meet-irreducible elements of $L / R$ not above $C l(x)$. The proof is available in "Lattice Differentials . . .".

A third source of differentials is the theory of finite lattices. Let $Q$ be an arbitrary finite lattice, let $A$ be the set of join-irreducible elements of $Q$ (those elements covering exactly one other element), and let $L$ be the Boolean algebra of all subsets of the set $A$. Define a closure operator $C l$ on the Boolean algebra $L$ by mapping every subset $x$ of the set $A$ into the subset $C l(x)$ containing all join-irreducible elements $e$ of $Q$ such that $e \leqslant s u p d, d \epsilon x$ the supremum being taken in the lattice $Q$. The differential of this closure operator is the structure differential of the lattice $Q$.

These three examples indicate the scope of the theory of differentials, a theory coextensive with the theories of finite lattices and of closure operators on finite modular lattices.

\section{Unit Increase Functions}

Before beginning a discussion of exact differentials, let us consider systems satisfying Whitney's first rank function axiom for matroids. Called unit increase functions, they provide a shortcut to exact differentials, a shortcut used in the single-element extension theorem. Differences of unit increase functions, like differentials, may be characterized by conditions on local graphs.

An integer-valued function $r$ defined on a finite modular lattice $L$ is a unit increase function if $r(y)$ $-r(x)$ is either 0 or 1 whenever an element $y$ covers an element $x$ in $L$. The function $R$ defined for all steps $[x, y]$ in $L$ by

$$
\begin{aligned}
R[x, y] & =1 \text { if } r(x)<r(y) \\
& =0 \text { otherwise }
\end{aligned}
$$

is the difference of the unit increase function $r$. Notice that for all steps $[x, y], R[x, y]=r(y)-r(x)$. In drawing comparisons with differentials, we think of the values 0 and 1 of $R$ as elements of the lattice $\{0,1\}$. The local characterization of differences of unit increase functions is as follows.

Proposition: Let L be a finite modular lattice. An assignment of values 0 and 1 to the steps of the lattice $\mathrm{L}$ is that of a difference of a unit increase function on L if and only if all local graphs are of the following five types: zero, mixed, prime, one or inverted. (See fig. 2).

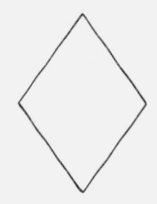

ZERO

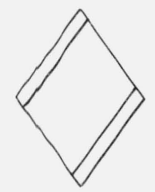

MIXED

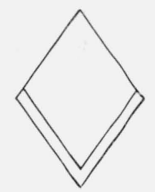

PRIME

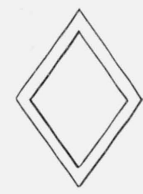

ONE

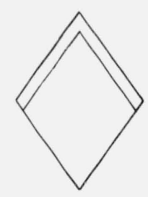

INVERTED
FIGURE 2

Proof: It is clear that the five possible local graphs not listed above may not appear in a difference of a unit increase function. Given an assignment $R$ of values 0 and 1 to the steps of $L$ such that all local graphs are zero, mixed, prime, one or inverted we construct a unit increase function $r$ by setting

$$
r(x)=\sum_{i=1}^{n} R\left[p_{i-1}, p_{i}\right], \text { where } p \text { is any path from } 0 \text { to } x .
$$

That $r(x)$ is independent of path is established by an inductive argument. Say $x$ is of height $n$ in the lattice $L$, and all paths of length $n-1$ or less have the same $R$-sum. Let $p: 0=p_{0}<p_{1}<\ldots<p_{n}=x$ and $q$ : $0=q_{0}<q_{1}<\ldots<q_{n}=x$ be two paths from 0 to $x$. If $p_{1}=q_{1}$, the $R$-sums are equal on $p$ and $q$. If $p_{1} \neq q_{1}$, $0=p_{1} \wedge q_{1}$ and $p_{1} \vee q_{1}$ covers $p_{1}$ and $q_{1}$. Form a path $s$ from $p_{1} \vee q_{1}$ to $x$. The $R$-sum along $p$ is equal to that to $p_{1}$ and $p_{1} \vee q_{1}$, thence along $s$, by the induction assumption. This sum is in turn equal to the sum to $q_{1}$ and $p_{1} \vee q_{1}$, thence along $s$, because the local graph on $0, p_{1}, q_{1}, p_{1} \vee q_{1}$ is one of the five types listed above. But this sum is equal to that along the path $q$, by the induction assumption. The $R$-sum, thus well-defined, is a unit increase function, because $r(y)-r(x)=R[x, y]$ $=0$ or 1 , for all steps $[x, y]$. 
This completes the local characterization of diferences of unit increase functions on finite modular lattices. A unit increase function whose difference has no inverted local graph is termed a Whitney rank function.

We find the following example due to Edmonds of some importance. Given a finite set, and a class of distinguished subsets called independent sets, a class closed with respect to taking of subsets, we may define the rank of a subset to be the number of elements in its largest independent subset. This rank is a unit increase function. The single assumption made, that every subset of an independent set is independent, is the assumption defining simplicial complexes in algebraic topology.

\section{Exact Differentials}

An exact differential was defined as a differential, all of whose local graphs are zero, mixed, prime, or one.

Proposition: Exact Differentials: If $\mathrm{r}$ is a unit increase function on a finite modular lattice and if the difference $\mathrm{R}$ of $\mathrm{r}$ is a differential, then $\mathrm{r}$ is a Whitney rank function, and $\mathrm{R}$ is an exact differential.

ProOF: If $r$ is a unit increase function with difference $R$, then all local graphs of $R$ are zero, mixed, prime, one, or inverted. If $R$ is also a differential, all local graphs are zero, mixed, prime, one, or inexact. Thus all local graphs are zero, mixed, prime, or one, $r$ is a Whitney rank function, and $R$ is an exact differential.

Proposition: Matroids: Let L be the Boolean algebra of all subsets of a finite set. Then an integervalued function $\mathrm{r}$ for which $\mathrm{r}(\Phi)=0$ is a Whitney rank function if and only if $\mathrm{r}$ is the rank function of a matroid.

Proof: Whitney's first axiom for matroids in terms of rank functions was taken as the definition of a unit increase function. If $r(x)=r(x \vee e)=r(x \vee f)$ for elements $e$ and $f$ not in a subset $x$, then $x, x \vee e$, $x \vee f, x \vee e \vee f$ form a local graph, on which $R[x, x \vee e]$ $=R[x, x \vee f]=0$. If $R$ is exact, this local graph must be zero, so $R[x \vee e, x \vee e \vee f]=R[x \vee f, x \vee e \vee f]=0$, and thus $r(x)=r(x \vee e \vee f)$, which gives Whitney's second axiom for matroids in terms of rank functions. Conversely, if $r$ is the rank function of a matroid, no local graph of its difference $R$ may be inverted, so $r$ is a Whitney rank function.

Several matroid theorems apply without alteration to exact differentials. For example, the statement "if an element $e$ is dependent upon a subset $x$ but upon no smaller subset, then $x$ is a circuit" may be phrased in terms of closed elements, as follows.

Proposition: Characterization of Meet-Irreducible Closed Elements: Given a step [x, y] in a modular lattice $\mathrm{L}$ and an exact differential $\mathrm{R}$ defined on $\mathrm{L}$, the element $\mathrm{x}$ is meet-irreducible in the lattice $\mathrm{L} / \mathrm{R}$ of closed elements if and only if the step $[\mathrm{x}, \mathrm{y}]$ is maximal, in the ordering on steps, among steps on which the differential $\mathrm{R}$ has value 1. (The proof may be found in "Lattice Differentials . . .".)

The concept of matroid duality is available for exact differentials. An element $x$ in the domain lattice
$L$ of a differential $R$ is dual-closed if $R[y, x]=0$ for all steps of the form $[y, x]$. The class of dual-closed elements is closed under the lattice operation sup; the image of this class in the inverted lattice $L$ is closed under the lattice operation inf, and gives rise to a closure operator and a differential $R^{*}$ on the inverted lattice $L$. It may be shown that $R^{* *}=R$ if $R$ is an exact differential.

Several conditions on differentials, equivalent to the exactness condition, will support the proof of the extension theorem. These conditions are given below; their equivalence is proven in "Lattice Differen. tials ...".

Proposition: Equivalent Exactness Conditions: If $\mathrm{R}$ is a differential on a finite modular lattice $\mathrm{L}$, then the following statements are equivalent.

(i) $\mathrm{R}$ is exact.

(ii) Closed Element Covering Property: If an element $\mathrm{y}$ covers an element $\mathrm{x}$ in the domain lattice $\mathrm{L}$, then the image of $\mathrm{Cl}(\mathrm{y})$ is equal to or covers the image of $\mathrm{Cl}(\mathrm{x})$ in the closed element lattice $\mathrm{L} / \mathrm{R}$.

(iii) Duality: $\mathrm{R} *[\tilde{\mathrm{y}}, \tilde{\mathrm{x}}]=1-\mathrm{R}[\mathrm{x}, \mathrm{y}]$ for all steps $[\mathrm{x}, \mathrm{y}]$.

(iv) Existence of Dual-Closed Elements: In every interval $[\mathrm{x}, \mathrm{z}]$ of the domain lattice on which $\mathrm{R}$ does not everywhere have the value 1 , there exists a relatively dual-closed element other than the element $\mathrm{x}$.

(v) Independence of Path: Differential sums of the form $\sum_{i=1}^{n} \mathrm{R}\left[\mathrm{p}_{\mathrm{i}-1}, \mathrm{p}_{\mathrm{i}}\right]$ along paths $\mathrm{p}: \mathrm{p}_{0}<\mathrm{p}_{1}<\ldots$ $<\mathrm{p}_{\mathrm{n}} \stackrel{i=1}{i=1}$ dependent only upon the end points $\mathrm{p}_{0}, \mathrm{p}_{n}$.

The closed element covering property of exact differentials, together with the construction of the closure operator $C l$ of a differential $R$, imply that, for any step $[x, y]$ of the domain lattice $L, C l(y)$ covers $C l(x)$ in $L / R$ if and only if $R[x, y]=1$, while $C l(y)=C l(x)$ if and only if $R[x, y]=0$. Conversely, if $p: x=p_{0}$ $<p_{1}<\ldots<p_{n}=z$ is any path from $x$ to $z$ in the domain lattice $L$ of an exact differential $R$, and if $C l(z)$ covers $C l(x)$ in the closed element lattice $L / R$, then $R\left[p_{0}, p_{1}\right]=1$ because $p_{0}=x$ is a closed element, and $R\left[p_{i-1}, p_{i}\right]=0$ for $i=2, . . ., n$, because $C l\left(p_{1}\right)$ and $C l\left(p_{n}\right)$ both cover $C l(x)$ in $L / R$, and must be equal.

Exactness of differentials is related to exactness of differential forms, in the theory of functions of several real variables. Given a differential $R$ on the Boolean algebra of a set $\left\{e_{1}, \ldots ., e_{n}\right\}$, and given a subset $x$ of this set, let

$$
M_{i}(x)=R\left[x, x \vee e_{i}\right]
$$

be the coefficient of $d e_{i}$ in the first order differential

$$
\sigma(x)=\sum_{i} M_{i}(x) d e_{i}
$$

Partial differentiation is defined by

$$
\frac{\partial M}{\partial e_{i}}(x)=M\left(x \vee e_{i}\right)-M(x)
$$

for any function $M$ from subsets to numbers. Then the usual exactness condition may be expressed in 
terms of the differential $R$ :

$$
\frac{\partial M_{i}}{\partial e_{j}}=\frac{\partial M_{j}}{\partial e_{i}}
$$

if and only if

$$
\begin{aligned}
& R\left[x \vee e_{j}, x \vee e_{i} \vee e_{j}\right]-R\left[x, x \vee e_{i}\right] \\
= & R\left[x \vee e_{i}, x \vee e_{j} \vee e_{i}\right]-R\left[x, x \vee e_{j}\right]
\end{aligned}
$$

if and only if

$$
\begin{aligned}
& R\left[x, x \vee e_{j}\right]+R\left[x \vee e_{j}, x \vee e_{i} \vee e_{j}\right] \\
= & R\left[x, x \vee e_{i}\right]+R\left[x \vee e_{i}, x \vee e_{i} \vee e_{j}\right],
\end{aligned}
$$

a statement which excludes inexact local graphs, yet is true on local graphs which are zero, mixed, prime, or one.

\section{Single-Element Extensions}

An understanding of the structure of the closed element lattice $L / R$ is important for the extension theorem. A lattice $Q$ is semimodular if, for any elements $x, y$ in $Q$ for which $x$ covers $x \wedge y$, it follows that $x \vee y$ covers $y$. A lattice $Q$ is geometric if it is semimodular and complemented, i.e., if all join-irreducible elements are atoms. In a geometric lattice, every element is expressible as a join of atoms and as a meet of coatoms.

Proposition: The Closed Element Lattice: If $\mathrm{R}$ is an exact differential on a finite modular lattice $\mathrm{L}$, the lattice $\mathrm{L} / \mathrm{R}$ is semimodular. If the domain lattice $\mathrm{L}$ is complemented, the lattice $\mathrm{L} / \mathrm{R}$ is geometric. (The proof may be found in "Lattice Differentials ....".)

Definition: A subset $J$ of the elements of a lattice is convex if $x \in J z \in J$, and $x<y<z$ imply $y \in J$. A cut of a lattice $Q$ is a convex subset of $Q$ which, if it is nonempty, contains the lattice element 1 . A cut $J$ of a lattice $Q$ is modular if $x \in J, y \in J$, and $x$ covers $x \wedge y$ imply $x \wedge y \in J$.

The concept of modular cut combines the properties of a Dedekind cut with a covering condition. A modular cut is not necessarily a lattice ideal: it is closed with respect to arbitrary multiplication ( $v$ ) by any lattice element, but must be closed with respect to sums $(\wedge)$ of elements in the cut only when one of the elements covers the sum. The simplest modular cuts in a lattice are the empty cut, the cut containing only the element 1 , and the cut containing the entire lattice. Any ideal $(x \in J, y \in J, z \in Q$ imply $x \vee z \in J$ and $x \wedge y \in J)$ is also a modular cut.

Theorem: Extensions Produce Modular Cuts: Let $\mathrm{R}$ be the differential of a matroid on the Boolean algebra $\mathrm{L}$ of all subsets of an $\mathrm{n}$-element set $\mathrm{X}$ : let $\mathrm{e}$ be any element of the set $\mathrm{X}$, and let $\mathrm{R}_{0}$ be the restriction of the matroid $\mathrm{R}$ to the Boolean algebra $\mathrm{L}_{0}$ of all subsets of the set $\mathrm{X}_{0}=\mathrm{X}-\{\mathrm{e}\}$. Let $\mathbf{J}$ be the set of all closed elements $\mathrm{x}$ of the matroid $\mathrm{R}_{0}$ such that $\mathrm{R}[\mathrm{x}, \mathrm{x} \vee \mathrm{e}]=0$.
Then $\mathrm{J}$ is a modular cut of the lattice $\mathrm{L}_{0} / \mathrm{R}_{0}$ of closed elements of the restricted matroid.

Proof: If the set $J$ is nonempty, and contains some element $x$ in the closed element lattice $L_{0} / R_{0}$, we have the ordering on steps $[x, x \vee e]<\left[1_{0}, 1\right]$, so the unit element $l_{0}$ of the lattice $L_{0} / R_{0}$ is also in the set $J . \quad J$ is convex, and therefore a cut, by the same argument: $x \in J, x \leqslant y$ imply $[x, x \vee e]<[y, y \vee e]$, so $R[x, x \vee e]=0$ implies $R[y, y \vee e]=0$ and $y \epsilon J$.

It remains to show that the cut $J$ of the lattice $L_{0} / R_{0}$ is modular. Assume closed subsets $x$ and $y$ in $L_{0}$ have an intersection $z$. Assume that, as elements of the closed element lattice $L_{0} / R_{0}, x$ and $y$ are in the cut $J$, and $x$ covers $z$, yet $z$ is not in the cut $J$. Choose a path $p: z=p_{0}<p_{1}<\ldots<p_{n}=x$ from $z$ to $x$ in the Boolean algebra $L . \quad R\left[p_{i-1}, p_{i}\right]=1$ only for $i=1$, because the image of $x$ covers the image of $z$ in the closed element lattice $L_{0} / R_{0}$. Since $x$ is in the cut $J, R[x, x \vee e]=0$, and the sum of the values of the differential $R$ from $z$ to $x \vee e$ is 1 . Lift the path $p$ to a path $p \vee e: \quad z \vee e=p_{0} \vee e<p_{1} \vee e<\ldots<p_{n} \vee e$ $=x \vee e$. Since $z$ is not in the cut $J, R[z, z \vee e]=1$, and $R$ has value 0 along the entire path $p \vee e$, because the $R$-sum is independent of path.

The subset $p_{1} \vee y$ covers $y$, and $R\left[y, p_{1} \vee y\right]=1$, because $y$ is closed relative to the restricted differential $R_{0}$. $R[y, y \vee e]=0$, because $y$ is in the cut $J$. Thus the local graph on $y, p_{1} \vee y, y \vee e, p_{1} \vee y \vee e$ is mixed, and $R\left[y \vee e, p_{1} \vee y \vee e\right]=1$. This contradicts the projective property of the differential $R$, because $[z \vee e$, $\left.p_{1} \vee e\right] \leqslant\left[y \vee e, p_{1} \vee y \vee e\right]$, yet $R$ has value 0 along the path $p \vee e$.

The converse of this theorem is also true, as we now prove.

Theorem: Modular Cuts Produce Extensions: Let $\mathrm{L}$ be the Boolean algebra of all subsets of an n-element set $\mathrm{X}$, and let $\mathrm{e}$ be any element of the set $\mathrm{X}$. Let $\mathrm{R}_{0}$ be any matroid on the Boolean algebra $\mathrm{L}_{0}$ of all subsets of the set $\mathrm{X}_{0}=\mathrm{X}-\{\mathrm{e}\}$, and let $\mathrm{J}$ be any modular cut of the closed element lattice $\mathrm{L}_{0} / \mathrm{R}_{0}$. There is a unique matroid $\mathrm{R}$ on the Boolean algebra $\mathrm{L}$, extending $\mathrm{R}_{0}$ and with the property, for all closed elements $\mathrm{x} \in \mathrm{L}_{0}$, $\mathrm{R}[x, x \vee \mathrm{e}]=0$ if any only if $\mathrm{x} \in \mathrm{J}$.

Proof: Let $r_{0}$ be the Whitney rank function of the matroid $R_{0} . \quad r_{0}(x)=\sum_{i} R_{0}\left[p_{i-1}, p_{i}\right]$ for any path $p$ from 0 to $x$ in the Boolean algebra $L_{0}$. Define a function $r$ on subsets in the Boolean algebra $L$ by: for every subset $x$ in $L_{0}$,

$$
\text { (i) } \begin{aligned}
r(x)=r_{0}(x) & \text { (ii) } \begin{aligned}
r(x \vee e) & =r_{0}(x)+1 \text { if } C l(x) \notin J \\
& =r_{0}(x) \text { if } C l(x) \in J .
\end{aligned}
\end{aligned}
$$

We prove that the function $r$ is a unit increase function. Steps in the Boolean algebra $L$ are of three types: $[x, y],[x, x \vee e]$, and $[x \vee e, y \vee e]$, where $[x, y]$ is a step in the Boolean algebra $L_{0}$. On steps of the first type, $r$ agrees with $r_{0}$, a unit increase function. On steps of the second type, we have defined $r(x \vee e)$ to be 
either equal to or one greater than $r(x)$. On steps of the third type, $r(y \vee e)-r(x \vee e)=r_{0}(y)-r_{0}(x)$ unless $C l(x) \notin J$ and $C l(y) \epsilon J$. But if $C l(x) \notin J$ and $C l(y) \epsilon J$, we know $C l(x)<C l(y)$, so $R_{0}[x, y]=1$, and $r(x \vee e)=$ $1+r_{0}(x)=r_{0}(y)=r(y \vee e)$. Thus $r$ is a unit increase function.

If $R$ is a matroid on the Boolean algebra $L$, and if $x$ is any subset of $X_{0}$, then $R[x, x \vee e]=R[C l(x), C l(\mathrm{x})$ $\vee e]$, because $R$ is subadditive on steps, and has value 0 on all steps of the interval $[x, C l(x)]$. Thus, if $R$ is a matroid on the Boolean algebra $L$, extending the matroid $R_{0}$ on the Boolean algebra $L_{0}$, and having the property, for all closed elements $x$ in $L_{0}$, that $R[x$, $x \vee e]=0$ if and only if the image of $x$ in $L_{0} / R_{0}$ is in the modular cut $J$, then the Whitney rank function of the matroid $R$ must be the unit increase function $r$ defined above. Thus there exists at most one matroid $R$ with the required properties. If the unit increase function $r$ can be shown to be a Whitney rank function, the existence of such a matroid $R$ extending $R_{0}$ is established.

Define the function $R$ on all steps of the Boolean algebra $L$ to be the difference of the unit increase function $r$. We must show that no local graph of $R$ on $L$ is inverted. The local graphs in $L$ are of three types:

(i) Local graphs on subsets $x \wedge y, x, y, x \vee y$, where neither $x$ nor $y$ contain the element $e$, but both cover $x \wedge y$.

(ii) Local graphs on subsets $x, y, x \vee e, y \vee e$, where $y$ covers $x$ and does not contain $e$.

(iii) Local graphs on subsets $z, x \vee z, y \vee z, x \vee y \vee z$, where $z=(x \wedge y) \vee e$ and the local graph on $x \wedge y, x, y$, $x \vee y$ is of type (i). No local graph of type (i) is inverted because $R_{0}$ is exact. No local graph of type (ii) is inverted, because $R[x, x \vee e]=0$ implies $C l(x) \epsilon J$, and $x<y$ implies $C l(y) \in J$ and $R[y, y \vee e]=0$.

If a local graph of type (iii) is inverted, the values of the difference $R$ are uniquely determined on the interval $[x \wedge y, x \vee y \vee z]$ of length 3. The local graph on $x \wedge y, x, y, x \vee y$ is one, by the projective property of $R$ across two local graphs of types (ii) and one of type (i), which are known to be either zero, mixed, prime, or one. The local graphs of type (ii) on $x \wedge y, y, z$, $y \vee z$ and on $x \wedge y, x, z, x \vee z$ are then prime, and that on $x, x \vee y, x \vee z, x \vee y \vee z$ is mixed. The resulting configuration is drawn in figure 3 . This configuration is

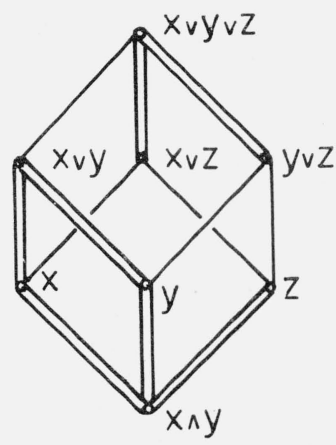

FiguRE 3 excluded by the modularity of the cut $J$, because the closures of $x$ and of $y$ cover the closure of $x \wedge y$ and are covered by the closure of $x \vee y$ in the closed element lattice $L_{0} / R_{0}$, while $R[x, x \vee z]=R[y, y \vee z]=0$ im plies $C l(x)$ and $C l(y)$ are in the modular cut $J$, and $R[x \wedge y, z]=1$ implies $C l(x \wedge y)$ is not in $J$.

\section{Linear Subclasses}

A preponderance of matroid theory is phrased in terms of circuits or bonds. Systematically, circuits and bonds are classes of distinguished subsets of a set, satisfying two axioms. In terms of closures, circuits are minimal nonempty dual-closed subsets. The circuits of the dual matroid (the bonds of the matroid) appear naturally as the set-complements of those closed subsets which are coatoms of the closed-set lattice.

Single-element extensions of matroids are readily characterized in terms of coatoms of the closed-set lattice. We show in this section how modular cuts are generated by linear subclasses ${ }^{5}$ of the set of coatomic closed subsets.

The passage from modular cuts to linear subclasses may be accomplished within the closed-set lattice. No reference is made to the manner in which this lattice is embedded in the domain of the matroid. Closed-set lattices of matroids are geometric; the structure differential of a finite geometric lattice is a matroid. The following theorems may thus be regarded as theorems about finite geometric lattices, and will be so written.

A linear subclass of the set $C$ of coatoms of a geometric lattice $Q$ is any subset $\mathscr{L}$ of the set $C$ which, if it contains two coatoms which cover their infimum, contains all other coatoms covering that same infimum.

The convex closure of a set $\mathscr{L}$ of coatoms of a geometric lattice $Q$ is the set of all lattice elements $x$ such that they and all lattice elements $y>x$ are expressible as an infimum of coatoms in the set $\mathscr{L}$.

Theorem: Generation of Modular Cuts: The convex closure of any subset $\mathscr{L}$ of the set of coatomic elements of a finite geometric lattice $\mathrm{Q}$ is a modular cut of the lattice $\mathrm{Q}$ if and only if the set $\mathscr{L}$ is a linear subclass. Every modular cut is the convex closure of the set of coatomic elements it contains.

Proof: Let $J$ be a nonempty modular cut of a finite geometric lattice $Q$. Every element of $J$ is expressible as an infimum of coatomic elements of $Q$, because $Q$ is geometric. All coatomic elements in such an expression are in the modular cut $J$, because $J$ is convex and contains the element 1 . Thus $J$ is contained in the convex closure of the set of coatomic elements in $J$. If an element $x$ of the lattice $Q$ is in the convex closure of the set of coatomic elements in $J$, choose a path $p: x=p_{0}<p_{1}<\ldots<p_{n}=1$ from $x$ to 1 . Since $p_{n-1}$ is expressible as an infimum of coatomic elements in $J$, yet is a coatomic element, $p_{n-1}$ is an element of $J$. Let $j$ be the largest index such that $p_{j} \notin J$, then find a coatomic element $z$ in $J$ such that $p_{j+1} \wedge z=p_{j}$. Since 
the cut $J$ is modular, $p_{j}$ is also in $J$. Thus the element $x$ is in the modular cut $J$, and every modular cut is the convex closure of the set of coatomic elements it contains.

Assume again that $J$ is a modular cut of a finite geometric lattice $Q$, and let $L$ be the set of coatomic elements in $J$. If $x, y$, and $z$ are coatomic elements of $Q$ such that $x$ and $y$ cover $x \wedge y$ and such that $x$ and $y$ are in the set $L$, then $x \wedge y$ is in the modular cut $J$. Since $J$ is convex, $z$ is also in $J$. Being coatomic, $z$ is in $\mathscr{L}$, and $\mathscr{L}$ is a linear subclass.

It remains to prove that the convex closure of any linear subclass is a modular cut. One step in this proof is of independent interest, and is set apart as a lemma.

Lemma: Covering Properties of Linear Subclasses: If $\mathscr{L}$ is a linear subclass of the coatomic elements of a finite geometric lattice $\mathrm{Q}$, and if $\mathrm{C}(\mathscr{L})$ is the convex closure of $\mathscr{L}$, then every lattice element $\mathrm{w} \notin \mathrm{C}(\mathscr{L})$ is covered by at most one element of $\mathrm{C}(\mathscr{L})$.

Proof: Let $w$ be a lattice element not in $C(\mathscr{L})$, yet covered by two distinct elements $u_{1}$ and $u_{2}$ of $C(\mathscr{L})$. Assume $w$ is maximal with respect to these properties. Since every element $x$ above $w$ is an infimum of coatomic elements above $w$, yet some element above $w$ is not an infimum of coatomic elements in the linear subclass $\mathscr{L}$, we may choose a coatomic element $z, w \leqslant z, z \notin L$. The rank $r(w)$ of the element $w$ in the lattice $Q$ is equal to neither $r(1)$ nor $r(1)-1$, because $w$ is covered by two distinct lattice elements. If $r(w)=r(1)-2, w$ is covered by two coatoms in the linear subclass $\mathscr{L}$, and thus must be in $C(\mathscr{L})$. Thus $r(w) \leqslant r(1)-3$.

Choose a path $p: w=p_{0}<p_{1} \ldots<p_{k}=z$ from $w$ to $z$, and let $q$ be any complement $\left(p_{1} \vee q=p_{2}\right.$ and $\left.p_{1} \wedge q=w\right)$ of $p_{1}$ in the lattice interval $\left[w, p_{2}\right]$ of height 2 . Note that no elements $p_{i}$ of the path $p$ are in $C(\mathscr{L})$, nor is $q$ in $C(\mathscr{L})$. We shall prove that either $p_{1}$ or $q$ is also covered by distinct elements of the convex closure $C(\mathscr{L})$, in contradiction to the maximality of $w$.

The elements $p_{1} \vee u_{1}, p_{1} \vee u_{2}$, and $u_{1} \vee u_{2}$ are either all distinct or all equal; they are all in $C(\mathscr{L})$ because they lie above either $u_{1}$ or $u_{2}$; none of them is equal to $p_{2}$. If they are distinct, $p_{1}$ is covered by $p_{1} \vee u_{1}$ and $p_{1} \vee u_{2}$, both in $C(\mathscr{L})$. If they are all equal, they are not equal to $p_{2}$, so the elements $q \vee p_{1}, q \vee u_{1}$, $q \vee u_{2}$ are all distinct. But $q \vee u_{1}$ and $q \vee u_{2}$ are in $C(\mathscr{L})$, and cover $q$.

The remainder of the proof of the theorem on generation of modular cuts now follows. Let $\mathscr{L}$ be a linear subclass of the set of coatoms of a finite geometric lattice $Q$, and let $C(\mathscr{L})$ be the convex closure of $\mathscr{L}$. The element 1 is in $C(\mathscr{L})$, because 1 is the infimum of the empty subset of $\mathscr{L}$. Convexity of $C(\mathscr{L})$ follows directly from the definition of convex closure, so $C(\mathscr{L})$ is a cut of the lattice $Q$. Assume $x$ and $y$ are in $C(\mathscr{L})$, and that $x$ covers $x \wedge y$. Choose a path $p: x \wedge y=p_{0}<p_{1}$ $<\ldots<p_{k}=y$ from $x \wedge y$ to $y$. Since $x$ covers $x \wedge y$, and $x \wedge y=x \wedge p_{i}(i=0,1, \ldots, k), x \vee p_{i}$ covers $p_{i}(i=0,1, \ldots, k)$. The elements $x \vee p_{i}$ are in $C(\mathscr{L})$, because $x \leqslant x \vee p_{i}$. Let $j$ be the least index such that $p_{j} \epsilon C(\mathscr{L})$. If $j \neq 0, p_{j-1}$ is covered by both $x \vee p_{j-1}$ and $p_{j}$, both of which are in $C(\mathscr{L})$. Applying the lemma on covering properties of linear subclasses, we see $p_{0}=$ $x \wedge y$ is in $C(\mathscr{L})$, verifying the modular property of the cut $C(\mathscr{L})$.

The characterization of modular cuts as convex closures of linear subclasses provides us with a description of single element extensions of matroids. If $R$ is a matroid defined on subsets of a set $X=X_{0} \cup\{e\}$, extending a matroid $R_{0}$ defined on subsets of the set $X_{0}$, then the element $e$ is dependent upon a subset $x$, i.e., $R[x, x \vee e]=0$, if and only if $C l(x)$ is in the convex closure of some linear subclass of the coatoms of $L_{0} / R_{0}$.

Let us now characterize the coatomic closed subsets of the extended matroid. We aim for a simple construction of the coatoms of an extended matroid, applicable to matrix-theoretic investigations.

Theorem: Closed Subsets of Single-Element Extensions: Let $\mathrm{R}$ be a matroid on the Boolean algebra $\mathrm{L}$ of all subsets of a finite set $\mathrm{X}=\mathrm{X}_{0} \cup\{\mathrm{e}\}$, which extends a matroid $\mathrm{R}_{0}$ on the Boolean algebra $\mathrm{L}_{0}$ of all subsets of the set $\mathrm{X}_{0}$, and which is produced by the modular cut $\mathrm{J}$ of the lattice $\mathrm{L}_{0} / \mathrm{R}_{0}$. Then the closed subsets of the matroid $\mathrm{R}$ are

(i) Closed subsets relative to $\mathrm{R}_{0}$ which are not in the modular cut $\mathrm{J}$,

(ii) Subsets of the form $\mathrm{x} v \mathrm{e}$ where $\mathrm{x}$ is a closed subset relative to $\mathrm{R}_{0}$ in the modular cut $\mathrm{J}$,

(iii) Subsets of the form $\mathrm{x} v \mathrm{e}$ where $\mathrm{x}$ is a closed subset relative to $\mathrm{R}_{0}$ not in the modular cut $\mathrm{J}$, and covered in the lattice $\mathrm{L}_{0} / \mathrm{R}_{0}$ only by elements not in the modular cut $\mathrm{J}$.

Proof: Subsets of $X$ not containing the element $e$ are closed relative to $R$ only if they are closed relative to $R_{0}$, and are not in the modular cut $J$. Subsets containing the element $e$ and closed relative to $R$ must, on deletion of $e$, become closed elements relative to $R_{0}$, because $R$ is projective on steps. To ascertain which subsets of the form $x \vee e$, for subsets $x$ closed relative to $R_{0}$, are closed relative to $R$, we observe that the $R$ value 1 on a step $[x, y]$ for $y \epsilon L_{0}$ decreases to 0 on the step $[x \vee e, y \vee e]$ if and only if the local graph on $x, y, x \vee e, y \vee e$ is prime. But this is true if and only if $C l(y)$, an element in the modular cut $J$, covers (in $\left.L_{0} / R_{0}\right) C l(x)$, an element not in $J$. The subset $x \vee e$ is closed relative to $R$ if and only if this occurs for no subset $y$ covering $x$.

The coatomic closed subsets of the extended matroid are easily picked out as those closed subsets $x$ such that paths $p$ from $x$ to 1 have $R$ value 1 only on the first step. They are those coatomic closed subsets of $X_{0}$ which are not in the linear subclass, together with subsets of the form $x \vee e, x$ being either a coatomic closed subset in the linear subclass, or a subset of rank $r(1)-2$ and covered in $L_{0} / R_{0}$ only by coatomic closed subsets not in the linear subclass.

Taking set-complements of coatomic closed subsets, we obtain a statement of the single-element extension theorem in terms of the bonds or circuits of a matroid. A linear subclass of a set of bonds is a set of bonds which contains, along with any pair $x, y$ of bonds whose union $x \cup y$ has rank 2 in the lattice 
of unions of bonds, any other bonds coniained in the union $x \cup y$. The word "circuit" may be substituted everywhere for "bond" in the previous sentence.

THEOREM: Bonds of Single-Element Extensions: Given a matroid with differential $\mathrm{R}_{0}$ on the Boolean algebra of subsets of a finite set $X_{0}$, and given a linear subclass $\mathscr{L}$ of the set $\mathrm{B}_{0}$ of bonds of $\mathrm{R}_{0}$, let $\mathrm{X}$ be the set $\mathrm{X}_{0} \cup\{e\}$. Then the following are the bonds of the matroid $\mathrm{R}$ produced by the linear subclass $\mathscr{L}$ :

(i) Bonds of $\mathrm{R}_{0}$ in the linear subclass $\mathscr{L}$;

(ii) Sets of the form $\mathrm{x} \cup\{\mathrm{e}\}$, where $\mathrm{x}$ is a bond of $\mathrm{R}_{0}$ outside the linear subclass $\mathscr{L}$;

(iii) Unions of bonds of $\mathrm{R}_{0}$, of rank 2 in the lattice of unions of bonds, and covering no bond in the linear subclass $\mathscr{L}$.

As a simple example, consider the bond matroid of a triangle with sides $a, b, c$, and an isthmus d attached at one of the vertices. The bonds are $a b, a c$, bc, and d. The single element extension corresponding to the linear subclass $\{a b\}$ has bonds (i) ab, (ii) ace, bce, de, (iii) acd, bcd. These are the bonds of the square with edges $a, b, e, d$ and a diagonal $c$ making a triangle with $\mathrm{a}$ and $\mathrm{b}$. The original graph is obtained by deleting the edge e.

In the above theorem and example, a matroid $R_{0}$ is extended to a matroid $R$ in such a way that $R_{0}$ is the restriction of $R$ to the Boolean algebra interval [0,1-e]. This restriction is Tutte's ${ }^{6} R_{0}=R \cdot X_{0}$, and is accomplished by "elimination" of the element $e$. Applying the above theorem to the dual matroid $R^{*}$ and interpreting the results in terms of the matroid $R$, we obtain a characterization of matroids $R$ such that $R_{0}$ is the restriction of $R$ to the Boolean algebra interval $[e, 1]$. This restriction is Tutte's $R_{0}=R \times X_{0}$, and is accomplished by "contraction" of the element $e$. Such extensions are produced by linear subclasses of the set $C_{0}$ of circuits of the matroid $R_{0}$; the circuits of the extended matroid are described in the above theorem, if "circuit" is substituted for "bond" throughout.

A class of unsolved problems suggested by Rota and Tutte is the characterization of those linear subclasses or modular cuts on matroids with property $P$, for which the associated single-element extension also has property $P$. This property $P$ may be binary, regular, even, graphic, or any property of the lattice of closed subsets. Characterizations of linear subclasses or modular cuts which preserve these properties may simplify inductive proofs of otherwise difficult theorems.

\section{Geometric Lattices}

The analysis of closed subsets of single-element extensions completed in the previous section, provides a method for the construction of all finite geometric lattices. Since every finite geometric lattice is the closed element of its structure differential, and since all matroids may be constructed inductively by modular cuts, all finite geometric lattices are con-

\footnotetext{
${ }_{6}$ W. T. Tutte, Trans. A.M.S., 1958.
}

structed by a sequence of modular cuts beginning with the simplest geometric lattice: that containing only the elements 0 and 1 .

Referring to the theorem on closed subsets of singleelement extensions, we see that the subsets of the first two types (those closed subsets $x \notin J$ and those closed subsets $x \vee e$ with $x \in J$ ) form a subsystem isomorphic to the closed-set lattice of the matroid $R_{0}$. Subsets of the third type must be added to the closedset lattice, and the appropriate covering lines must be added to the lattice diagram, as follows.

Given a finite geometric lattice $Q$ and any modular cut $J$ of $Q$, let Ext $J$ be composed of those elements not in $J$, and covered by no elements of $J$. A new lattice $Q^{\prime}$ may be defined, extending $Q$, by adding to the lattice $Q$ an element $x^{\prime}$ corresponding to each element $x$ in Ext $J$, and by extending the covering relation " $y$ covers $x$ " to include elements $x$ and $y$ of $Q^{\prime}$ such that

(i) $x, y$ are in $Q$, and $y$ covers $x$ in $Q$

(ii) $y=x^{\prime}$

(iii) $x=z^{\prime}, y \in J$, and $[z, y]$ is an interval of length 2 in the lattice $Q$.

This inductive procedure is carried out for four steps in figure 4.

Although any modular cut yields an extension of the structure differential of a geometric lattice $Q$, only those modular cuts $J$ for which Ext $J$ is nonempty change the lattice $Q$. The set Ext $J$ is nonempty, and thus the extension is nontrivial on $Q$, if and only if no atom of the lattice $Q$ is in the modular cut $J$.

\section{Numerical Classification of Matroids}

We have seen the application of the single-element extension theorem to the construction of finite geometric lattices. Let us now turn our attention to a numerical classification of matroids. We prove the existence, on any $n$-element set, of at least one matroid in each of $2^{n}$ classes, and thereby obtain a lower bound on the number of different matroids on a given set.

The sequence of values of the differential of a matroid on the steps of a path from 0 to $l$ in the domain lattice may be thought of as forming a word in a language employing two letters, the letter 0 coming before the letter 1 in the alphabet. Of the words thus associated with a given matroid the word coming first in alphabetical order is an isomorphy invariant of the matroid, and serves as an index for a classification system.

We use the term least path to indicate a path, from 0 to 1 in the domain lattice of a matroid, if the word formed of the differential values along this path is alphabetically the first among all such words associated with the same matroid. The word associated with a least path of a matroid we shall call the first word of the matroid.

Given any $n$-letter word formed of the letters 0 and 1 , we shall prove the existence of a matroid having that word as first word. This establishes the exist- 


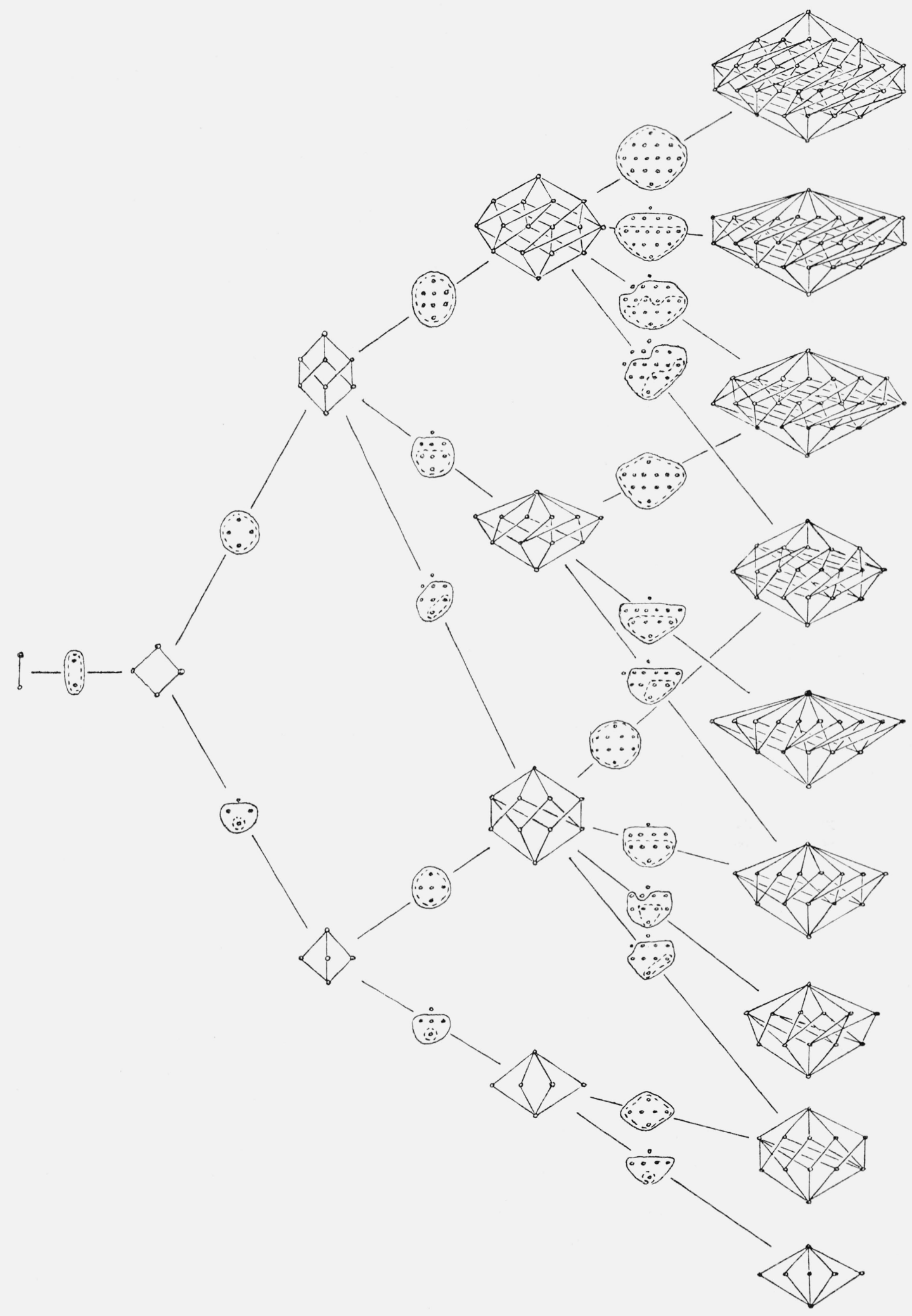

FIGURE 4. Geometric lattices with $\mathrm{n}$ atoms, $\mathrm{n}-1,2,3,4,5$.

Modular cuts generating each extension are indicated on the connecting lines. The set $c J$ is marked with a solid line; the set Ext $J$ with a dotted line. 
ence of at least $2^{n}$ isomorphically inequivalent matroids defined on the Boolean algebra of subsets of an $n$ element set. The existence proof proceeds by induction, and utilizes our knowledge of single-element extensions.

THEOREM: Existence of Matroids With A Given First Word: Let $\mathrm{W}$ be any $\mathrm{n}$-letter word, i.e., any sequence of length $\mathrm{n}$ consisting of zeros and ones. There exists a matroid $\mathrm{R}$ on a Boolean algebra $\mathrm{L}$ of all subsets of an $\mathrm{n}$-element set, such that the word $\mathrm{W}$ is the first word of the matroid $\mathrm{R}$ on $\mathrm{L}$.

Proof: The theorem is obvious for $n=1$. Assume we are given a word $W=w_{1}, \ldots ., w_{n}$ of length $n$, and that for any word of length $n-1$ there exists a matroid defined on a Boolean algebra of all subsets of an $(n-1)$ element set, such that the word of length $n-1$ is the first word. If $w_{n}=1$, find a matroid $R_{0}$ on the interval $[0,1-e]$, for which $w_{1}, \ldots, w_{n-1}$ is first word. Choose the empty modular cut, and let $R$ be the matroid produced by that cut. Then $R[x, x \vee e]=1$ for all subsets $x$ in the lattice interval $[0,1-e]$, and $R[x \vee e, y \vee e]=$ $R[x, y]$ for all pairs $x, y$ of elements in the lattice interval $[0,1-e]$. That $W$ is the first word for $R$ follows from the fact that the word for a path employing a step greater than $[0, e]$ in any position but the last may be obtained from the corresponding word for the projection of this path into the sublattice $[0,1-e]$ by insertion of the letter 1 at some point, moving all later letters back one space. This results in a word at least as late as $W$ in the alphabetical ordering.

If the final letter $w_{n}$ in the word $W$ is 0 , the proof is a bit more intricate. Form a matroid $R_{0}$ on the Boolean algebra interval $[0,1-e]$, having $w_{1}, \ldots ., w_{n-1}$ as first word. On the lattice of closed subsets $[0,1-e] /$ $R_{0}$, let a modular cut $J$ contain only the closed subset $1-e$ of $R_{0}$. Construct an extended matroid $R$ on the Boolean algebra $L$ of all subsets of the $n$-element set in accordance with the extension theorem. The coatomic closed subsets of the resulting differential $R$ are the coatomic closed subsets of $R_{0}$ on the interval $[0,1-e]$, together with those subsets of the form $x \vee e$, where $x$ is a closed element of $L_{0} / R_{0}$ of rank $r(1)-2$, because no coatoms of $L_{0} / R_{0}$ are in the modular cut $J$.

If a subset $x$ in the interval $[0,1-e]$ is not connected to $1-e$ by a path along which $R_{0}=0$, it is contained in some coatomic closed subset of $R_{0}$ not in the modular cut $J$, so $R[x, x \vee e]=1$. In any path from 0 to $x \vee e$ for any such set $x$, the projection of this path into the lattice interval $[0,1-e]$, then via the step $[x, x \vee e]$, is a lesser path. Let $p$ be a least path for the extended matroid $R$, and one which passes through a minimum number of subsets containing the element $e$. By the argument just given, if the subset $p_{k}$ is the first subset containing the element $e$ occurring in the path $p$, the subset $p_{k-1}$ either must be a coatomic closed subset relative to the matroid $R_{0}$, or must be connected to $1-e$ by a path along which $R_{0}=0$. If the subset $p_{k-1}$ is connected to $1-e$ by a path along which $R_{0}=0$, all steps above $p_{k-1}$ in the path $p$ have $R$ value zero, so the path following $p$ to $p_{k-1}$, then via $1-e$ to 1 gives rise to the same word, and involves the element $e$ in fewer subsets. On the other hand, if $p_{k-1}$ is a coatomic closed subset of $R_{0}$, the step $\left[p_{k-1}, p_{k}\right]$ is the final step of $p$ for which $R$ has value one. Any path along $p$ to $p_{k-1}$, then via $1-e$ to 1 gives rise to the same word, and involves the element $e$ in fewer subsets. Thus the path $p$ passes through $1-e$, and is a least path. The restriction of $p$ to the interval $[0,1-e]$ is a least path for $R_{0}$, so must give rise to the word $w_{1}$, . . ., $w_{n-1}$. Since $w_{n}$ is 0 , the word for the least path $p$ of the matroid $R$ is the word $W$.

CoRollary: A Lower Bound for the Number of Matroids: There are at least $2^{\mathrm{n}}$ nonisomorphic matroids on a Boolean algebra of all subsets of an n-element set.

PRoof: There are $2^{n}$ different words of length $n$, and each is the first word for some matroid.

The construction of a matroid with a given first word may be carried out methodically. On a diagram of the Boolean algebra of all subsets of an $n$-element set, choose any path to be the least path, and indicate on it the letters of the given first word. Then mark all steps according to the projective and subadditive properties of differentials, the requirement that every local graph be zero, mixed, prime or one, and the requirement that the given path be least.

When all the implications of the first word are exhausted, a matroid may not be fully determined. A matroid can then be defined in more than one way with the given first word; the number of such ways we shall term the multiplicity of the word.

DEFINITION: The multiplicity $\theta(W)$ of a word $W$ of length $n$ is the number of isomorphically inequivalent matroids with first word $W$, definable on the Boolean algebra of all subsets of an $n$-element set.

We have proven that all words have multiplicity $\theta$ at least equal to one. All words of one, two, or three letters have multiplicity equal to one. A single four letter word, 1010, has multiplicity two.

It is interesting to speculate about what additional isomorphy invariants may serve to classify matroids. The set of matroids with a given first word may be arranged in a partially ordered system, along paths of which the complexity ${ }^{7}$ (the number of bases in the matroid) increases. This investigation, which is involved with the dichromate (see footnote 7) of a matroid, is the subject of a future paper.

\section{Bibliography}

Birkhoff, G. (1935), Abstract linear dependence and lattices, Amer. J. 57, 800-804.

Crapo, H. H., Lattice differentials, and the theory of combinatorial independence. (to appear).

Dilworth, R. P. (1941), The arithmetical theory of Birkhoff lattices, Duke J. 8, 286-299.

- (1944), Dependence relations in a semi-modular lattice, Duke J. 11, 575-587.

Edmonds, J., et al. (Sept. 1964), Seminar: The Mathematical Theory of Matroids, National Bureau of Standards.

MacLane, S. (1938), A lattice formulation for transcendence degrees and $p$-bases, Duke J. 4, 455-468.

\footnotetext{
W. T. Tutte, Can. J., 1954.
} 
Ore, O. (1962), Theory of Graphs, A.M.S. Colloquium Publications 38.

Rado, R. (1942), A Theorem on independence functions, Quart. J. of Math. (Oxford) 13, 83-89.

Note on Independence Functions, Proc. Lond. Math. Soc. (3) $7,300-320$ (1957).

Rota, G.-C. (1964), On the foundations of combinatorial theory, Zeitschr. fur Wahrscheinlichkeitstheorie 2, 340-368.

Szasz, G., Introduction to Lattice Theory (Academic Press, New York, 1963).
Tutte, W. T. (1954), A Contribution to the theory of chromatic polynomials, Can. J. 6, 80-91.

- A Class of Abelian Groups, Can. J. 8, 13-28 (1956).

A homotopy theorem for matroids, I-II, Trans. A.M.S. 88, 144-174 (1958).

Matroids and Graphs, Trans. A.M.S. 90, 527-552 (1959).

Whitney, H. (1935), On the abstract properties of linear dependence, Amer. J., 5 7, 509-533.

(Paper 69B1-133) 